\title{
A CAS-System for Enhancements in Hip Surgery
}

\author{
Langlotz F., Stucki M., Nolte L.-P., Ganz R."
}

\begin{abstract}
M. E. Müller Institute for Biomechanics, University of Berne, CH-3010 Berne, Switzerland
"Department of Orthopaedic Surgery, University of Berne, CH-3010 Berne, Switzerland
\end{abstract}

\section{INTRODUCTION}

Osteotomies of the pelvis, in particular of the acetabulum, are means to correct dysplasia of the hip joint. Different surgical techniques have been presented to liberate the acetabulum and move it into a position that grants better coverage of the femoral head $[1,2,5]$. These difficult surgeries mainly rely on the experience and the imagination of the surgeon since many steps of the intervention have to be performed without direct visual control on the used instruments. Computer Assisted Orthopaedic Surgery (CAOS) has proven its potential to guide in difficult approaches in other fields of surgery $[4,6]$. The present work introduces a system for intraoperative image navigation in periacetabular osteotomy surgeries. Infra-red light emitting diodes (LEDs) mounded onto various osteotomes and onto the iliac crest are tracked by an optoelectronic camera system. Our CAOS-System is based on preoperative CT scans and allows for real-time visualization of surgical tools in various $2 \mathrm{D}$ and $3 \mathrm{D}$ reconstructions of the image data.

\section{DESCRIPTION OF THE SYSTEM}

Preoperatively three modified $3.5 \mathrm{~mm}$ cortical screws are inserted into the pelvic rim to provide easy to identify fiducial markers for the intraoperative paired points matching algorithm. A series of $5 \mathrm{~mm}$ spaced CT scans is taken. The raw image data is loaded into a graphics computer (Sun Microsystems, Eschenbach, Switzerland) and a visualized in three perpendicular cuts. Preoperative planning includes the definition of matching points represented by the inserted screws and additional landmark points. If desired, a segmentation for $3 D$ visualization can be performed.

Intraoperatively a standard surgical approach as described in [2] is performed. In order to be tracked by an optoelectronic space digitizer, the OPTOTRAK 3020 (Northern Digital, Waterloo, Canada), all tools are instrumented with marker probes holding four LEDs (see Figure 1). A similar probe, the so-called dynamic reference base (DRB), is attached to the iliac crest enabling compensation of patient motion. This probe consists of a small base part which is rigidly fixed to the bone and a handy snap-on-carrier that may be easily removed and re-attached during surgery (see Figure 2). This may be required when relocating the patient or due to spatial limitations.

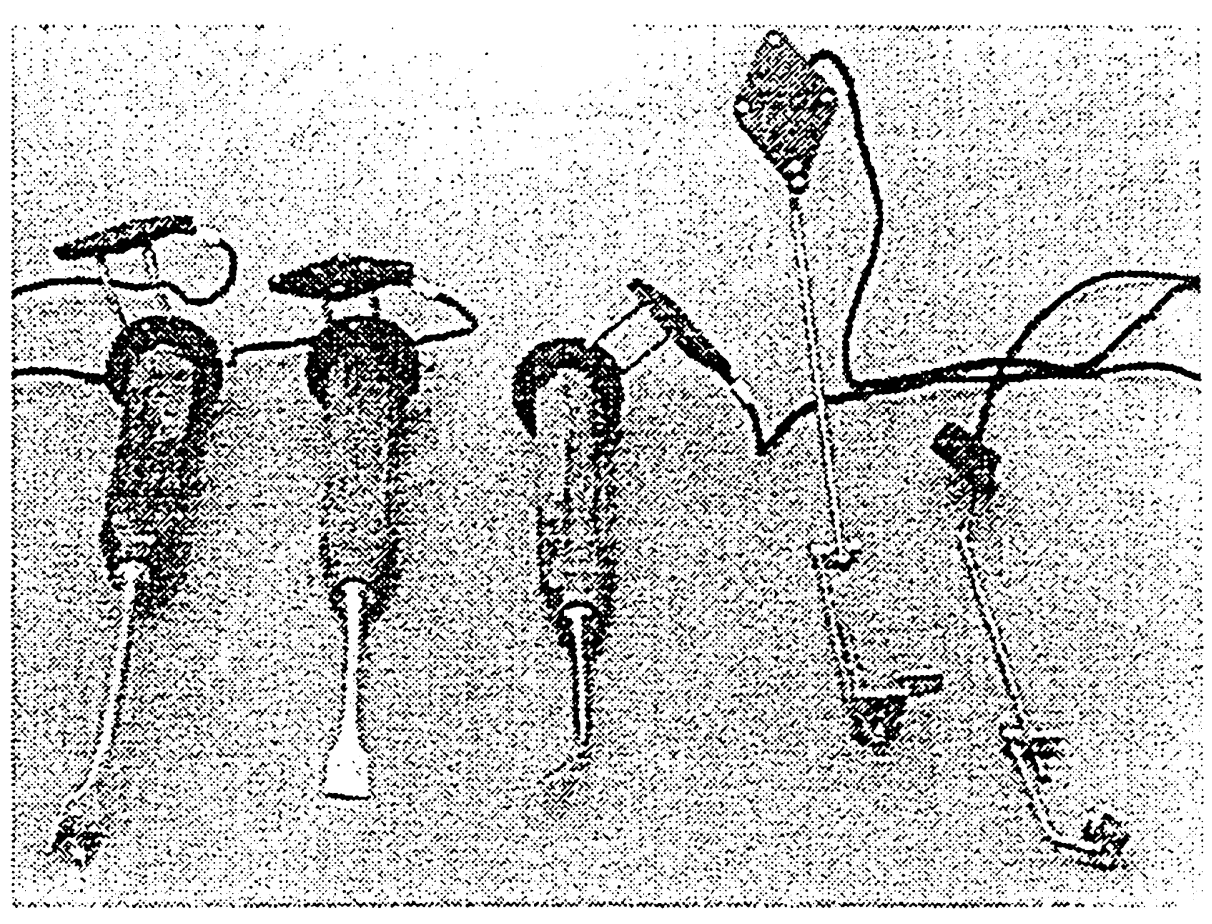

Figure I: Instrumented tool set for $P A O$

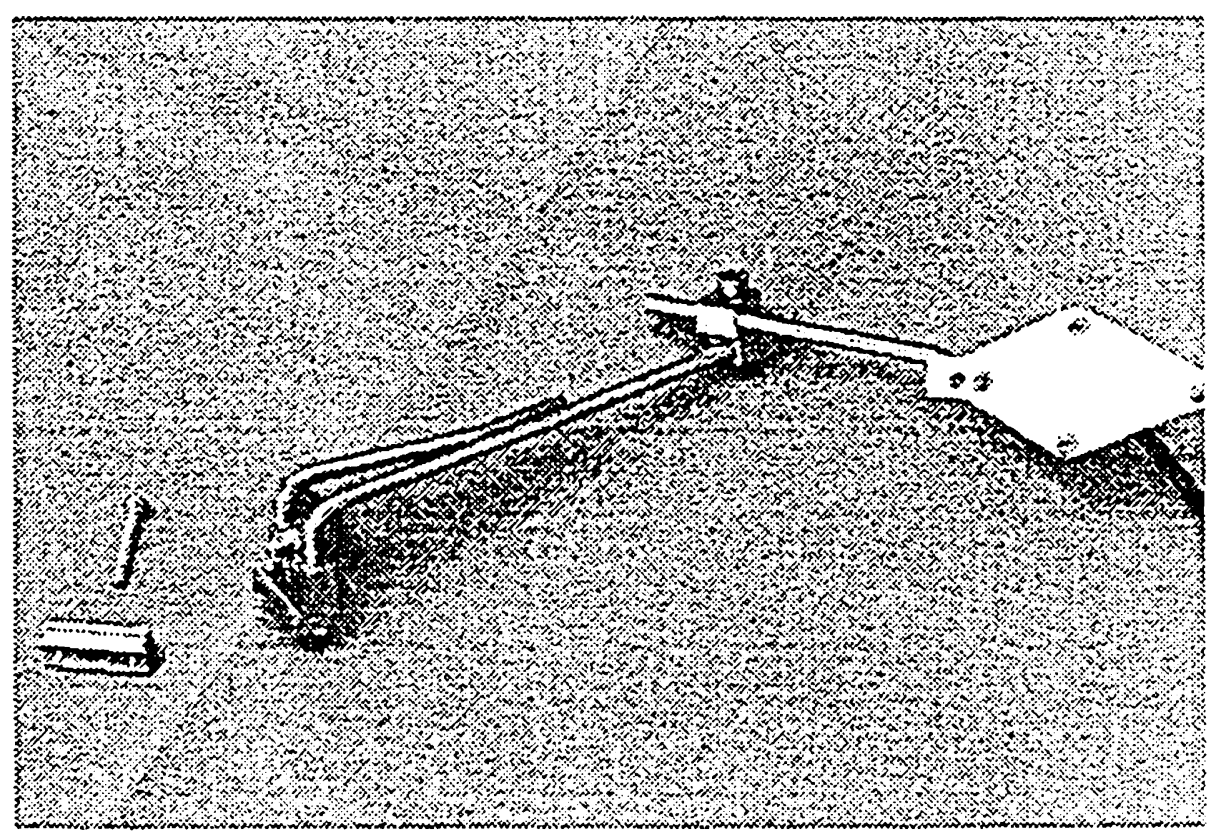

Figure 2: Two-part construction of Dynamic Reference Base

The matching or registration of the CT image with the patient's anatomy is performed by digitizing the preoperatively defined matching points on the patient. The matching algorithm then finds the coordinate transformation between patient space and image space. Once this link has been established, it is possible to display any tracked tool at its current position in the CT image in real-time. Different kinds of presentation have been evaluated to find optimal display modes with a maximum of readability. In general, static and dynamic views may be distinguished: Static views. show a nonmoving CT image with the surgical tool overlaid as 
graphical object. This may be a planar section through or maximum intensity projections of the image volume. 3D scenes based on preoperative segmentation data are seen as static views as well. Dynamic views change their contents according to the location and orientation of the instrument to be followed. Examples are sections always aligned with the instrument's axis or 3D scenes with an automatically re-sizing cutout giving an insight to the instrument's tip. For PAO we found a combination of static and dynamic $2 D$ and $3 D$ views most informative and readable. Figure 3 shows an intraoperative computer screen with two maximum intensity projections, a 3D reconstruction and 5 planar sections aligned with the osteotome.

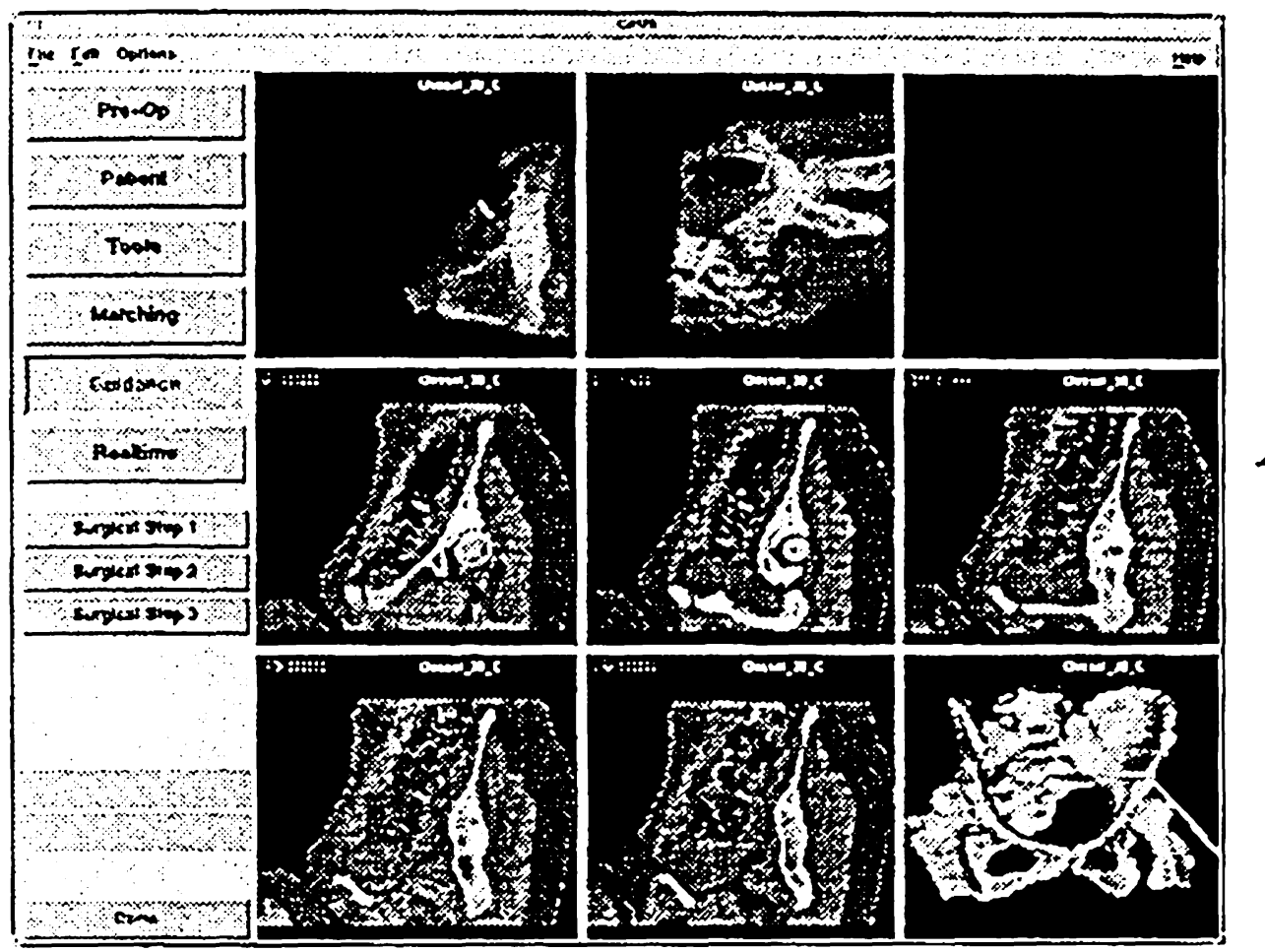

Figure 3: Intraoperative screen-shot showing static and dynamic views

For a controlled reorientation of the acetabular fragment an LED-probe similar to the DRB is fixed to the acetabulum before it is completely liberated. When moving the fragment its actual displacement is shown with the respect to its original location and orientation. This allows for precise application of surgical planning data [3].

Special attention has been paid to find a user interface that allows for the safe control of the system in a clinical environment. All functions may be selected by either a foot switch or a virtual keyboard that can be sterilized. This keyboard is a function plate with label fields representing options in the software. Whenever any tool is pointing to one of these fields, the associated action is performed. To enable the usage of different tool sets and individual tool configurations, every tool plug that connects the LEDs to the tracking device houses an EEPROM chip. This chip stores information like the tool name, its class, and calibration data. An additional software handles the tool set and detects changes in the actual tool configuration allowing for intraoperative change of instruments.

\section{RESULTS}

Since November 1995, the presented system for computer assisted hip surgery has been successfully used during six cases of periacetabular osteotomy so far. It has proven its potential to give exact feed-back of the surgical action being performed. This may reduce the risk to damage sensitive structures in this difficult intervention. The user interface and the possibility to control the system by the medical staff only has received positive echo. The matching algorithm was found to be accurate and reliable. However, the strategy will be reworked to overcome the need for an additional operation prior to the main intervention. New surface matching algorithms are currently under development.

\section{DISCUSSION}

Our CAOS system has jumped over the first barriers in hip surgery. Additional work effort has to be spent to make the segmentation and matching procedure more comfortable. The principles of CAOS will open gates towards new techniques in orthopaedic surgery in the next years. Minimally invasive approaches will benefit from the accuracy and navigation power provided by these systems.

\section{REFERENCES}

[1] K. Chiari: Ergebnisse mit der Beckoosteotomie als Pfannendachplastik. Z. Orthop. 87:14-26 (1955)

[2] R. Ganz, K. Klaue, T. S. Vinh, J. W. Mast: A new periacetabular osteotomy for the treatment of hip dysplasias. Clin. Orthop. 232:26-36 (1988)

[3] K. Klaue, A. Wallin, R. Ganz: CT evaluation of the hip prior to osteotomy. Clin Orthop 232:15-25, 1988.

[4] L.-P. Nolte, L. Zamorano, H. Visarius, U. Berlemann, F. Langlotz, E. Arm, O. Schwarzenbach: Clinical evaluation of a system for precision enhancement in spine surgery. Clin. Biomech. 10:293-303 (1995)

[5] R. B. Salter: Innominate osteotomy in the treatment of congenital dislocation and subluxation of the hip. J. Bone Joint. Surg. 43B:518-539 (1961)

[6] L. Zamorano, Z. Jiang, A. M. Kadi: Computerassisted neurosurgery system: Wayne State University hardware and software configuration. Comp. Med. Imaging Graph. 18:257-271 (1994) 